\title{
Survey on Matrix Factorization Using Information Fusion
}

\author{
Rutuja Mane $^{1}$, A. N. Bandal ${ }^{2}$ \\ ${ }^{1,2}$ Department of Computer Engineering, SIT, Savitribai Phule, Pune University, Pune, India
}

\begin{abstract}
Information sets that portray the watched framework from different points of view and record the conduct of its individual parts, find that issues in science and Engineering. Heterogeneous information sets can be all things considered mined by information combination. Fusion can concentrate on a particular target connection and endeavor specifically related information together with logical information and information about framework's requirements. The paper describe that this paper portray an data fusion approach with punished matrix tri-factorization (DFMF) that at the same time factorizes information grids to uncover shrouded affiliations. The methodology can specifically consider any information that can be communicated in a framework, counting those from highlight based representations, ontologies, affiliations and systems. This paper exhibit the utility of DFMF for quality capacity forecast undertaking with eleven diverse information sources and for expectation of pharmacologic activities by combining six information sources. Our information combination calculation thinks about positively to option information reconciliation approaches and accomplishes higher exactness than can be gotten from any single information source alone.
\end{abstract}

Keywords: Information fusion, intermediate data integration, matrix factorization, data mining, bioinformatics, cheminformatics

\section{Introduction}

Information possesses large amounts of all regions of human attempt. This paper might assemble different information sets that are specifically identified with the issue, or information sets that are inexactly identified with our study in any case, could be helpful when joined with other information sets. Consider, for instance, the exposome that incorporates the totality of human attempt in the investigation of infection. Let us say that we analyze helplessness to a specific illness furthermore, have admittance to the patients' clinical information together with information on their demographics, propensities, living situations, companions, relatives, motion picture watching propensities, and film sort philosophy. Mining such a differing information accumulation may uncover fascinating examples that would stay shrouded on the off chance that we would investigate just specifically related, clinical information. What if the illness was less basic in living zones with more open spaces or in situations where individuals need to walk rather than drive to the closest staple?

Routines for information combination can aggregately treat information sets what's more; consolidate different information sources notwithstanding when they contrast in their calculated, logical and typographical representation. Singular information sets may be fragmented, yet as a result of their differences and complementarily, combination can enhance the heartiness and prescient execution of the coming about models.

As per Pavlidis et al. (2002), data fusion methodologies can be arranged into three fundamental classifications contingent upon the demonstrating stage at which combination takes place. Early (or full) combination changes all information sources into a solitary element based table and regards this as a solitary information set that can be investigated by any of the well established highlight based machine learning calculations. The derived models can on a basic level incorporate any sort of connections between the elements from inside and between the information sources. Early mix depends on methods for highlight development. For our exposome illustration, persistent particular information would need to incorporate both clinical information and data from the motion picture classification ontologies. The previous may be insignificant as this information is as of now identified with every particular patient, while the last requires more perplexing component building. Early joining too dismisses the measured structure of the information.

In late (choice) joining, every information source offers ascend to a different model. Forecasts of these models are combined by model weighting. Once more, preceding model derivation, it is essential to change every information set to encode relations to the target idea. For our sample, data on the film inclinations of companions and relatives should be mapped to illness affiliations. Such changes might not be paltry and should be created freely for each information source.

The most youthful branch of information combination calculations is halfway (fractional) combination. Calculations in this class unequivocally address the assortment of information and wire them through induction of a solitary joint model. Moderate combination does not combine the input information, nor does it create separate models for every information source. It rather holds the structure of the information sources by consolidating it inside of the structure of prescient model. This specific methodology is frequently favored in view of its prevalent prescient precision however for a given model sort, it requires the improvement of another deduction calculation.

We here report on the improvement of another technique for middle of the road information combination taking into account obliged grid factorization. Our point was to develop a calculation that requires no or just negligible change of info information what's more, can breaker highlight based representations, ontologies, affiliations and systems. We 


\section{International Journal of Science and Research (IJSR) \\ ISSN (Online): 2319-7064 \\ Index Copernicus Value (2013): 6.14 | Impact Factor (2014): 5.611}

concentrate on the test of managing accumulations of heterogeneous information sources, keeping in mind demonstrating that our technique can be utilized on sizable issues from ebb and flow examination, scaling is not the center of the present paper.

\section{Related Work}

In paper [1] author describ an data fusion approach with penalized matrix tri-factorization (DFMF) that at the same time factorizes data matrices to reveal hidden associations. The methodology can specifically consider any information that can be expressed in a matrix, including those from feature-based representations, ontologies, associations and networks.

In this[2] paper that a more exact meaning of the field of data combination can be of advantage to analysts inside of the field, who may utilize such a definition when motivating their own work and assessing the commitment of others. In addition, it can empower analysts and experts outside the field to additional effortlessly relate their own work to the field and all the more effectively comprehend the systems' extent and techniques created in the field. Past meanings of data combination are checked on from that point of view, counting meanings of information and sensor combination, and their suitability as definitions for the whole exploration field are examined. Taking into account qualities and shortcomings of existing definitions, a novel definition is proposed, which is contended to successfully satisfy the necessities that can be put on a meaning of data combination as a field of examination.

In numerous areas [3] there will exist distinctive representations or "perspectives" portraying the same arrangement of items. Taken alone, these perspectives will frequently be lacking or fragmented. Consequently a key issue for exploratory information examination is the mix of numerous perspectives to find the basic structures in a space. This issue is made more troublesome when difference exists between perspectives. Author present another unsupervised calculation for consolidating data from related perspectives, utilizing a late mix technique. Combination is performed by applying a methodology in light of grid factorization to gathering related bunches delivered on individual views. This yields a unique's projection bunches in the structure of another arrangement of "meta-bunches" covering the whole space. We likewise give a novel model determination system for recognizing the right number of Meta-bunches. Assessments performed on various multi-view content grouping issues show the calculation's adequacy.

In this [4] paper depicts a computational system for coordinating and drawing inferences from a gathering of far reaching estimations. Each dataset is represented via a kernel function, which characterizes summed up similitude connections between sets of substances, for example, qualities or proteins. The kernel representation is both adaptable and proficient, and can be connected to a wide range of sorts of information. Besides, portion capacities got from diverse sorts of information can be joined in a clear manner. Late advances in the hypothesis of portion routines have given proficient calculations to perform such mixes in a manner that minimizes a measurable misfortune capacity. These routines misuse semidefinite programming strategies to diminish the issue of discovering streamlining portion mixes to a raised streamlining issue.

Computational analyses performed utilizing yeast genome wide datasets, including amino corrosive arrangements, hydropathy profiles, quality expression information and known protein-protein connections, show the utility of this methodology. A factual taking in calculation prepared from these information to perceive specific classes of proteinslayer proteins what's more, ribosomal proteins-performs fundamentally superior to the same calculation prepared on any single kind of information.

In their [5] endeavors to comprehend cell capacity at the sub-atomic level, we must have the capacity to integrate data from divergent sorts of genomic information. We consider the issue of deducing quality utilitarian classications from a heterogeneous information set comprising of DNA microarray expression estimations and phylogenetic profiles from entire genome succession examinations. We exhibit the application of the support vector machine (SVM) learning calculation to this useful deduction errand. Our outcomes propose the significance of misusing former information's about the data heterogeneity. Specifically, we propose a SVM kernel method that is expressly heterogeneous. Likewise, we portray highlight scaling strategies for further giving so as to misuse earlier information of heterogeneity every information sort distinctive weights.

In chart based [6] learning models, substances are frequently spoken to as vertices in an undirected chart with weighted edges portraying the connections between substances. In numerous true applications, on the other hand, elements are frequently related with relations of distinctive sorts and/or from diverse sources, which can be very much caught by various undirected diagrams over the same arrangement of vertices. Step by step instructions to endeavor such different sources of data to improve surmisings on elements remain a fascinating open issue. In this paper, we concentrate on the issue of bunching the vertices in light of different charts in both unsupervised and semi-regulated settings. As one of our commitments, we propose Linked Matrix Factorization (LMF) as a novel method for combining data from different chart sources. In LMF, every chart is approximated by lattice factorization with a chart particular variable and an element basic to all diagrams, where the basic variable gives elements to all vertices. Investigates SIAM diary information demonstrate that (1) we can enhance the grouping precision through intertwining various wellsprings of data with a few models, and (2) LMF yields better or aggressive results analyzed than other diagram based bunching techniques.

The late years [7] have seen a surge of hobbies of semiregulated bunching strategies, which intend to cluster the information set under the direction of some supervisory data. Generally those supervisory information takes the type of pair wise imperatives that indicate the similitude/uniqueness between the two focuses. In this paper, we propose a novel lattice factorization based methodology for semi-regulated bunching. In addition, we extend our calculation to co-bunch 


\section{International Journal of Science and Research (IJSR) \\ ISSN (Online): 2319-7064 \\ Index Copernicus Value (2013): 6.14 | Impact Factor (2014): 5.611}

the information sets of different sorts with limitations. At last the experiments on UCI information sets and genuine Bulletin Board Systems (BBS) information sets demonstrate the prevalence of our proposed system.

In this [8] paper that Non-negative matrix factorization (NMF) has already been appeared to be a helpful deterioration for multivariate information. Two diverse multiplicative calculations for NMF are broke down. They vary just marginally in the multiplicative variable utilized as a part of the overhaul rules. One calculation can be appeared to minimize the customary slightest squares lapse while the other minimizes the summed up Kullback-Leibler difference. The monotonic meeting of both calculations can be demonstrated utilizing a helper capacity practically equivalent to that utilized for demonstrating meeting of the Expectation-Amplification calculation. The calculations can likewise be deciphered as corner to corner rescaled slope drop, where the rescaling variable is ideally decided to guarantee union.

It is surely [9] understood that great instatements can enhance the velocity and precision of the arrangements of numerous nonnegative lattice factorization (NMF) calculations. Numerous NMF calculations are delicate as for the instatement of $\mathrm{W}$ or $\mathrm{H}$ or both. This is particularly valid for calculations of the exchanging minimum squares (ALS) sort, including the two new ALS calculations that we exhibit in this paper. We look at the aftereffects of six introduction strategies (two standards and four new) on our ALS calculations. In conclusion, we talk about the handy issue of picking a fitting union paradigm.

In this [10] paper that we introduce a bound together perspective of matrix factorization that edges the distinctions among famous routines, for example, NMF, Weighted SVD, E-PCA, MMMF, pLSI, pLSI-pHITS, Bregman co-grouping, and numerous others, as far as a little number of demonstrating decisions. Large portions of these approaches can be seen as minimizing a summed up Bregman disparity, what's more, we demonstrate that (i) a direct substituting projection calculation can be connected to any model in our brought together view; (ii) the Hessian for every projection has exceptional structure that makes a Newton projection possible, notwithstanding when there are correspondence requirements on the components, which takes into consideration framework co-grouping; and (iii) substituting projections can be summed up to at the same time consider an arrangement of grids that share dimensions. These perceptions instantly yield new enhancement calculations for the above factorization routines, and propose novel speculations of these routines, for example, fusing line and section predispositions, and including on the other hand unwinding grouping requirements.

[11] Fusing various data sources can yield critical advantages to effectively achieve learning assignments. Numerous studies have focused on fusing data in regulated learning contexts. We display a methodology to use different data sources as closeness information for unsupervised learning. Taking into account closeness data, the grouping undertaking is expressed as a non-negative grid factorization issue of a blend of similitude estimations. The tradeoff between the education of information sources and the inadequacy of their blend is controlled by an entropy-based weighting system. With the end goal of model determination, a strength based methodology is utilized to guarantee the determination of the most self-reliable speculation. The tests show the execution of the strategy on toy and in addition genuine information sets.

In this [12] Paper that due to the high false positive rate in the high-throughput test routines to find protein communications, computational techniques are essential and vital to finish the interactome quickly. On the other hand, when building arrangement models to recognize putative protein communications, contrasted with the undeniable decision of positive tests from genuinely collaborating protein sets, it is normally difficult to select negative specimens, on the grounds that non-collaborating protein sets allude to those right now without trial or computational confirmation to bolster a physical cooperation or an utilitarian affiliation, which, however, could associate as a general rule. To handle this trouble, rather than utilizing heuristics as in numerous current works, in this paper we unravel it in a principled path by figuring the protein association forecast issue from a new numerical point of view of perspective - inadequate framework finish, and propose a novel Non negative Matrix Tri-Factorization (NMTF) based network culmination way to deal with foresee new protein associations from existing protein collaboration systems. Since network fulfillment just requires positive specimens yet not utilize negative examples, the test in existing order based systems for protein collaboration forecast is dodged. Through utilizing complex regularization, we further create our strategy to coordinate distinctive natural information sources, for example, protein groupings, quality expressions, protein structure data, and so forth.

Social learning [13] is turning out to be progressively vital in numerous ranges of use. Here, we present a novel way to deal with social learning taking into account the factorization of a three way tensor. We demonstrate that dissimilar to other tensor methodologies, our technique has the capacity perform aggregate learning through the latent parts of the model and give a proficient calculation to process the factorization. We substantiate our hypothetical contemplations with respect to aggregate learning abilities of our model by the method for tests on both another dataset and a dataset ordinarily utilized in element determination. Moreover, we appear on normal benchmark datasets that our methodology accomplishes better or on-par results, if contrasted with current cutting edge social learning arrangements, while it is essentially speedier to process.

A few high-throughput strategies, for instance, yeast twohybrid framework and mass spectrometry technique, can decide protein interactions, which, suffer from high falsepositive rates. Besides, numerous protein collaborations anticipated by one technique are not upheld by another. In this way, computational strategies are important and vital to finish the interactome speedily. In this work [14], they figure the issue of foreseeing protein interactions from new mathematical perspective-sparse matrix completion, and propose a novel nonnegative matrix factorization (NMF) 


\section{International Journal of Science and Research (IJSR) \\ ISSN (Online): 2319-7064 \\ Index Copernicus Value (2013): 6.14 | Impact Factor (2014): 5.611}

based network fulfillment way to deal predict new protein interactions from existing protein interaction networks. Through utilizing complex regularization, they facilitate add to our technique to incorporate distinctive organic information sources, for example, protein sequences, gene expressions, protein structure data, and so forth.

In this paper [15], author propose a machine learning based technique, module-guided Random Forests (mgRF), to integrate genotypic and gene expression information to explore genetic factors and molecular component hidden complex characteristics. mgRF is an expanded Random Forests strategy upgraded by a network analysis for recognizing numerous correlated variables of different types. They connected mgRF to genetic markers and gene expression information from a cohort of F2 female mouse intercross. mgRF beat a few existing strategies in their broad examination. Their new approach has an enhanced execution when combining both genotypic and gene expression information contrasted with utilizing both of the two types of information alone.

In the hierarchy of data [16], information and knowledge, computational techniques assume a major role in the initial processing of information to concentrate data, however only they turn out to be less powerful to aggregate knowledge from data. The Kyoto Encyclopedia of Genes and Genomes (KEGG) asset has been produced as a kind of perspective learning base to help this last process. Specifically, the KEGG pathway maps are generally utilized for biological understanding of genome sequences and other highthroughput information. The link from genomes to pathways is made through the KEGG Orthology framework, a gathering of manually characterized ortholog groups distinguished by $K$ numbers. To better automate this interpretation process the KEGG modules characterized by Boolean articulations of $\mathrm{K}$ numbers have been extended and moved forward.

\section{Comparative Analysis}

According to previous studies, where they provide data matrix as a product of low-rank matrix factors that are found by solving an optimization problem. This paper classifies and describes these requirements.

Table 1: Comparison Table

\begin{tabular}{|c|c|c|c|c|c|c|c|c|c|c|}
\hline Methods & {$[\mathbf{1}]$} & {$[\mathbf{3}]$} & {$[\mathbf{6}]$} & {$[\mathbf{7}]$} & {$[\mathbf{8}]$} & {$[\mathbf{9 ]}$} & {$[\mathbf{1 0}]$} & {$[\mathbf{1 1}]$} & {$[\mathbf{1 2}]$} & {$[\mathbf{1 3}]$} \\
\hline Non-Negative Matrix Factorization & Yes & Yes & No & No & Yes & Yes & Yes & Yes & Yes & No \\
\hline Joint Matrix Factorization & No & No & No & No & No & No & No & No & No & Yes \\
\hline Tri factorization & Yes & No & No & Yes & No & No & No & No & Yes & No \\
\hline Linked Matrix Factorization & No & No & Yes & No & No & No & No & No & No & No \\
\hline Novel Matrix Factorization & No & No & No & Yes & No & No & No & No & No & No \\
\hline
\end{tabular}

\section{Conclusion}

The paper survey that another matrix factorization data fusion combination calculation called DFMF. The methodology is adaptable what's more, as opposed to cutting edge kernel based routines, requires negligible, if any, preprocessing of information. This recent element, the capacity to show multi-social what's more, multi-item sort information, and DFMF's magnificent exactness what's more, time reaction, are the central focal points of our new calculation.

DFMF can display any gathering of information sets, each of which can be communicated as a grid. Assignments from bioinformatics also, cheminformatics considered here that were customarily viewed as order issues embody only one sort of information mining issues that can be tended to with our strategy. We expect the utility of factorization based information combination in multi-assignment learning, affiliation mining, grouping, connection expectation or organized yield forecast.

\section{References}

[1] Marinka Zitnik and Blaz Zupan, “Data Fusion by Matrix Factorization", IEEE transactions on attern analysis and machine intelligence, vol. 37, no. 1, january 2015.

[2] H. Bostrom, S. F. Andler, M. Brohede, R. Johansson, A. Karlsson, J.vanLaere, L. Niklasson, M. Nilsson, A.
Persson, and T. Ziemke, - n the definition of information fusion as a field of research,'Univ. Skovde, School Humanities Informat, Skovde, Sweden,Tech. Rep. HS-IKI-TR-07-006, 2007.

[3] D. Greene and P. Cunningham, -Amatrix factorization approach for integrating multiple data views," in Proc. Eur. Conf. Mach. Learn. Knowl. Discovery Databases, 2009, pp. 423-438.

[4] G. R. G. Lanckriet, T. De Bie, N. Cristianini, M. I. Jordan, and W. S. Noble, - Astatistical framework for genomic data fusion," Bioinformat., vol. 20, no. 16, pp. 2626-2635, 2004.

[5] P. Pavlidis, J. Cai, J. Weston, and W. S. Noble, - Earning gene functional classifications from multiple data types," J. Comput. Biol., vol. 9, pp. 401-411, 2002.

[6] W. Tang, Z. Lu, and I. S. Dhillon, -Gilstering with multiple graphs," in Proc. IEEE 9th Int. Conf. Data Mining, 2009, pp. 1016-1021.

[7] F. Wang, T. Li, and C. Zhang, -Smi-supervised clustering via matrix factorization," in Proc. SIAM Int. Conf. Data Mining, 2008, pp. 1-12.

[8] D. D. Lee and H. S. Seung, -Agorithms for nonnegative matrix factorization," in Advances in Neural Information Processing Systems, T. K. Leen, T. G. Dietterich, and V. Tresp, Eds., Cambridge, MA, USA: MIT Press, 2000, pp. 556-562.

[9] R. Albright, J. Cox, D. Duling, A. N. Langville, and C. D. Meyer, -Algrithms, initializations, and convergence for the nonnegative matrix factorization," Dept. Math., 


\section{International Journal of Science and Research (IJSR) \\ ISSN (Online): 2319-7064}

Index Copernicus Value (2013): 6.14 | Impact Factor (2014): 5.611

North Carolina State University, Raleigh, NC, USA, Tech. Rep. 81706, 2006.

[10] A. P. Singh and G. J. Gordon, - Aunified view of matrix factorization models," in Proc. Eur. Conf. Mach. Learn. Knowl. Discovery Databases, 2008, pp. 358-373.

[11] T. Lange and J. M. Buhmann, -Fision of similarity data in clustering," in Proc. Adv. Neural Inf. Process. Syst., 2005, pp. 723-730.

[12] H. Wang, H. Huang, C. H. Q. Ding, and F. Nie, Predicting protein-protein interactions from multimodal biological data sources via nonnegative matrix tri-factorization," in Res. Comput.Molecular Biol., vol. 7262, pp. 314-325, 2012.

[13] M. Nickel, - Athree-way model for collective learning on multirelational data," in Proc. 28th Int. Conf. Mach. Learn., 2011, pp. 809-816.

[14] H. Wang, H. Huang, C. H. Q. Ding, and F. Nie, Predicting protein-protein interactions from multimodal biological data sources via nonnegative matrix tri-factorization," in Res. Comput. Molecular Biol., vol. 7262, pp. 314-325, 2012.

[15]Z. Chen and W. Zhang, Integrative analysis using module guided random forests reveals correlated genetic factors related to mouse weight," PLoS Comput. Biol., vol. 9, no. 3, p. e1002956, 2013.

[16] M. Kanehisa, S. Goto, Y. Sato, M. Kawashima, M. Furumichi, and M. Tanabe, Đata, information, knowledge and principle: Back to metabolism in kegg," Nucleic Acids Res., vol. 42, no. D1, pp. D199-D205, 2014. 